\title{
Survey of Clinical Laboratory Practices for 2015 Middle East Respiratory Syndrome Coronavirus Outbreak in the Republic of Korea
}

Mi-Kyung Lee, M.D. ${ }^{1}$, Sinyoung Kim, M.D. ${ }^{2}$, Mi-Na Kim, M.D. ${ }^{3}$, Oh Joo Kweon, M.D. ${ }^{1}$, Yong Kwan Lim, M.D. ${ }^{1}$, Chang-Seok Ki, M.D. ${ }^{4}$, Jae-Seok Kim, M.D. ${ }^{5}$, Moon-Woo Seong, M.D. ${ }^{6}$, Heungsup Sung, M.D. ${ }^{4}$, Dongeun Yong, M.D. ${ }^{2}$, Hyukmin Lee, M.D. ${ }^{7}$, Jong-Rak Choi, M.D. ${ }^{2}$, Jeong-Ho Kim, M.D. ${ }^{2}$; MERS-CoV Laboratory Response Task Force of The Korean Society for Laboratory Medicine

Department of Laboratory Medicine ${ }^{1}$, Chung-Ang University College of Medicine, Seoul; Department of Laboratory Medicine², Yonsei University College of Medicine, Seoul; Department of Laboratory Medicine ${ }^{3}$, Asan Medical Center, University of Ulsan College of Medicine, Seoul; Department of Laboratory Medicine and Genetics ${ }^{4}$, Samsung Medical Center, Sungkyunkwan University School of Medicine, Seoul; Department of Laboratory Medicine ${ }^{5}$, Kangdong Sacred Heart Hospital, Hallym University College of Medicine, Seoul; Department of Laboratory Medicine ${ }^{6}$, Seoul National University College of Medicine, Seoul; Department of Laboratory Medicine ${ }^{7}$, Catholic Kwandong University College of Medicine, Incheon, Korea

Background: It is crucial to understand the current status of clinical laboratory practices for the largest outbreak of Middle East respiratory syndrome coronavirus (MERS-CoV) infections in the Republic of Korea to be well prepared for future emerging infectious diseases.

Methods: We conducted a survey of 49 clinical laboratories in medical institutions and referral medical laboratories. A short questionnaire to survey clinical laboratory practices relating to MERS-CoV diagnostic testing was sent by email to the directors and clinical pathologists in charge of the clinical laboratories performing MERS-CoV testing. The survey focused on testing volume, reporting of results, resources, and laboratory safety.

Results: A total of 40 clinical laboratories responded to the survey. A total of 27,009 MERS-CoV real-time reverse transcription PCR (rRT-PCR) tests were performed. Most of the specimens were sputum (73.5\%). The median turnaround time (TAT) was $5.29 \mathrm{hr}$ (first and third quartile, 4.11 and $7.48 \mathrm{hr}$ ) in 26 medical institutions. The median TAT of more than a half of the laboratories (57.7\%) was less than $6 \mathrm{hr}$. Many laboratories were able to perform tests throughout the whole week. Laboratory biosafety preparedness included class II biosafety cabinets (100\%); separated pre-PCR, PCR, and post-PCR rooms (88.6\%); negative pressure pretreatment rooms (48.6\%); and negative pressure sputum collection rooms (20.0\%).

Conclusions: Clinical laboratories were able to quickly expand their diagnostic capacity in response to the 2015 MERS-CoV outbreak. Our results show that clinical laboratories play an important role in the maintenance and enhancement of laboratory response in preparation for future emerging infections.

Key Words: Middle East respiratory syndrome coronavirus (MERS-CoV), Korea, Outbreak, Clinical laboratory, Preparedness, Survey
Received: August 11, 2015

Revision received: September 3, 2015

Accepted: November 10, 2015

Corresponding author: Jeong-Ho Kim Department of Laboratory Medicine, Yonsei University College of Medicine, 50-1 Yonsei-ro, Seodaemun-gu, Seoul 03722, Korea

Tel: +82-2-2228-2448

Fax: +82-2-364-1583

E-mail: jeongho@yuhs.ac

(c) The Korean Society for Laboratory Medicine This is an Open Access article distributed under the terms of the Creative Commons Attribution Non-Commercial License (http://creativecommons.org/licenses/by-nc/3.0) which permits unrestricted non-commercial use, distribution, and reproduction in any medium, provided the original work is properly cited.

\section{INTRODUCTION}

On May 20, 2015, Middle East respiratory syndrome coronavirus
(MERS-CoV) was confirmed for the first time in an infected patient in the Republic of Korea. Although the primary case traveled to the United Arab Emirates and Saudi Arabia, the patient 
did not initially report his recent travel in those countries [1]. This case led to transmission of MERS-CoV both within a hospital and between hospitals and eventually resulted in the largest outbreak of MERS-CoV infections outside the Arabian Peninsula.

At that time, we had limited information on MERS-CoV, and only a few clinical laboratories were prepared to perform molecular diagnostic testing for the virus. During the longer than two months of the outbreak, 186 confirmed cases were diagnosed by real-time reverse transcription PCR (rRT-PCR) of MERS-CoV, and specimens from tens of thousands of suspected cases, including individuals who contacted the confirmed cases, were submitted for this testing. Many clinical laboratories were instructed to set up facilities to perform MERS-CoV rRT-PCR on site in a short time to fight against the transmission of this virus in their own institutes.

An earlier outbreak in 2009 of a novel strain of H1N1 influenza virus A (H1N1 influenza) affected laboratories worldwide, with a potentially tremendous impact on the practices of clinical laboratories [2]. The outbreak of H1N1 influenza also had a great influence in the Republic of Korea. The field of molecular testing for pathogens has been expanded in clinical laboratories, and the molecular testing industry has responded quickly with the production of new molecular test kits. Numerous studies, including viral etiology, epidemiology, risk factors, clinical and laboratory characteristics, and diagnostic tests, have been reported [3]. Despite the fact that clinical laboratory practice was a critical element in the response to the H1N1 influenza outbreak, there are only a few reports regarding this aspect of testing $[2,4,5]$.

The ability of clinical laboratories to respond appropriately to an outbreak is significant in pathogen control. Therefore, it is crucial to understand the current status of clinical laboratories in the Republic of Korea in order to be well prepared for any future emerging infectious diseases. In this article, we present the results of a survey of clinical laboratory practices during the 2015 MERS-CoV outbreak.

\section{METHODS}

The study population consisted of clinical laboratories performing diagnostic testing for MERS-CoV in medical institutions (hospitals and medical centers) and referral medical laboratories among clinical laboratories accredited by the Korean Laboratory Accreditation Program [6].

This survey was an initiative of the MERS-CoV Laboratory Response Task Force of the Korean Society for Laboratory Medi- cine. We conducted a survey of 49 clinical laboratories. A short questionnaire to assess clinical laboratory practices related to MERS-CoV diagnostic testing was sent by email to the directors and the clinical pathologists (laboratory physicians) in charge of the clinical laboratories performing MERS-CoV tests. The survey focused on the number of tests and the number of positive test results for MERS-CoV, turnaround time (TAT), request process, collection and transportation of specimens, testing and reporting, communication of results, laboratory safety, and reagents and equipment.

\section{RESULTS}

A total of 40 clinical laboratories (81.6\%, 40/49), including 35 medical institutions and 5 referral medical laboratories, responded to the survey. The number of beds in the medical institutions was as follows: $<500$ beds, 3 institutions (8.6\%); 5001,000 beds, 20 (57.1\%); and >1,000 beds, 12 (34.3\%). All clinical laboratories used rRT-PCR as the detection method for MERS-CoV.

\section{Testing volume and specimen type}

The number of MERS-CoV rRT-PCR tests performed was collected from 32 medical institutions and five referral medical laboratories. Data up to July 15, 2015 were collected according to the day and specimen type. A total of 27,009 MERS-CoV rRTPCR tests were performed at 32 medical institutions ( $N=$ $11,502)$ and five referral medical laboratories $(N=15,507)$ (Table 1 and Fig. 1). The proportion of medical institutions was significantly underestimated because one tertiary care hospital submitted responses for the survey but not the specimen list, and the numbers of MERS-CoV rRT-PCR tests and positive specimens at this institution would have been predominant in the reporting medical institutions. MERS-CoV rRT-PCR testing at all medical institutions and referral medical laboratories increased dramatically in early June (Fig. 1). The number of clinical laboratories that initiated MERS-CoV testing increased in the first two to three weeks (Fig. 2). Daily test volumes peaked on June 24 (1,088 tests) and began to decrease thereafter (Fig. 1). Among the 27,009 MERS-CoV rRT-PCR specimens, 246 (0.9\%) and $91(0.3 \%)$ specimens were positive and indeterminate, respectively (153 and 71 specimens at medical institutions, 93 and 20 specimens at referral medical laboratories; Table 1).

Most of the specimens for MERS-CoV rRT-PCR were sputum (73.5\%). A total of $74.7 \%$ of all specimens tested and $82.9 \%$ of positive specimens were specimens from the lower respiratory 
Table 1. Number of MERS-CoV tests and positive and indeterminate results according to the specimen type

\begin{tabular}{|c|c|c|c|c|c|c|c|c|c|}
\hline \multirow[b]{2}{*}{ Specimen type } & \multicolumn{3}{|c|}{ Total } & \multicolumn{3}{|c|}{ Medical institution $(\mathrm{N}=32)$} & \multicolumn{3}{|c|}{ Referral medical laboratories $(\mathrm{N}=5)$} \\
\hline & $\begin{array}{l}N \text { of tests } \\
(\%)\end{array}$ & $\begin{array}{l}\mathrm{N} \text { of positive } \\
\text { tests (\%) }\end{array}$ & $\begin{array}{c}\mathrm{N} \text { of } \\
\text { indeterminate } \\
\text { tests }(\%)\end{array}$ & $\begin{array}{l}N \text { of tests } \\
(\%)\end{array}$ & $\begin{array}{l}N \text { of positive } \\
\text { tests (\%) }\end{array}$ & $\begin{array}{c}\mathrm{N} \text { of } \\
\text { indeterminate } \\
\text { tests (\%) }\end{array}$ & $\begin{array}{l}N \text { of tests } \\
(\%)\end{array}$ & $\begin{array}{c}\mathrm{N} \text { of positive } \\
\text { tests (\%) }\end{array}$ & $\begin{array}{c}\mathrm{N} \text { of } \\
\text { indeterminate } \\
\text { tests (\%) }\end{array}$ \\
\hline Sputum & $19,843(73.5)$ & $178(0.90)$ & $72(0.36)$ & 7,304 (63.5) & $97(1.33)$ & $56(0.77)$ & $12,539(80.9)$ & $81(0.65)$ & $16(0.13)$ \\
\hline $\begin{array}{l}\text { Bronchoscopy } \\
\text { specimens* }\end{array}$ & $202(0.7)$ & $11(5.45)$ & $0(0.0)$ & $69(0.6)$ & $11(15.94)$ & $0(0.0)$ & $133(0.9)$ & $0(0.0)$ & $0(0.0)$ \\
\hline Tracheal aspirates & $138(0.5)$ & $15(10.87)$ & $1(0.72)$ & $135(1.2)$ & $15(11.11)$ & $1(0.74)$ & $3(0.0)$ & $0(0.0)$ & $0(0.0)$ \\
\hline $\begin{array}{l}\text { Nasopharyngeal } \\
\text { aspirates }\end{array}$ & $293(1.1)$ & $0(0.0)$ & $0(0.0)$ & $233(2.0)$ & $0(0.0)$ & $0(0.0)$ & $60(0.4)$ & $0(0.0)$ & $0(0.0)$ \\
\hline $\begin{array}{l}\text { Nasopharyngeal \& } \\
\text { oropharyngeal swab }\end{array}$ & $2,240(8.3)$ & $21(0.94)$ & $14(0.63)$ & $1,216(10.6)$ & $20(1.64)$ & $13(0.70)$ & $1,024(6.6)$ & $1(0.10)$ & $1(0.10)$ \\
\hline Nasopharyngeal swab & $3,150(11.6)$ & $6(0.09)$ & $3(0.10)$ & 2,398 (20.8) & $6(0.25)$ & $0(0.0)$ & $752(4.8)$ & $0(0.0)$ & $3(0.40)$ \\
\hline Oropharyngeal swab & $1,075(4.0)$ & $14(1.30)$ & $0(0.0)$ & $79(0.7)$ & $3(3.80)$ & $0(0.0)$ & $996(6.4)$ & $11(1.10)$ & $0(0.0)$ \\
\hline Others (stool, blood) & $68(0.3)$ & $1(1.47)$ & $1(1.47)$ & $68(0.6)$ & $1^{\dagger}(1.47)$ & $1^{\ddagger}(1.47)$ & $0(0.0)$ & $0(0.0)$ & $0(0.0)$ \\
\hline Total & $27,009(100.0)$ & $246(0.91)$ & $91(0.34)$ & $11,502(100.0)$ & $153(1.33)$ & $71(0.62)$ & $15,507(100.0)$ & $93(0.60)$ & $20(0.13)$ \\
\hline
\end{tabular}

*Bronchoscopy specimens include specimens obtained from bronchoalveolar lavage (BAL) and bronchial washing (BW); ${ }^{\circ B l o o d ;}{ }^{~} S$ Stool. Abbreviation: MERS-CoV, Middle East respiratory syndrome coronavirus.



Fig. 1. Number of Middle East respiratory syndrome coronavirus (MERS-CoV) tests performed in Korea from May 28 to July 15.

tract, such as sputum, bronchoscopy specimens, or tracheal aspirates (Table 1). All nasopharyngeal aspirates $(\mathrm{N}=293)$ were negative for MERS-CoV rRT-PCR.

\section{Turnaround time}

The TAT is defined as the time from the receipt of specimens in the laboratory to the reporting of the results. Unfortunately, 35\% of the laboratories (nine medical institutions and five referral medical laboratories) were not able to provide or analyze data on TAT.

The median TAT was $5.29 \mathrm{hr}$ (first and third quartile, 4.11 and $7.48 \mathrm{hr}$ ) for 26 medical institutions, and the minimum and maximum median TAT (first and third quartile) were $4.00 \mathrm{hr}$ (3.35 and $5.10 \mathrm{hr}$ ) and $26.46 \mathrm{hr}$ (19.59 and $31.36 \mathrm{hr}$ ), respec- 


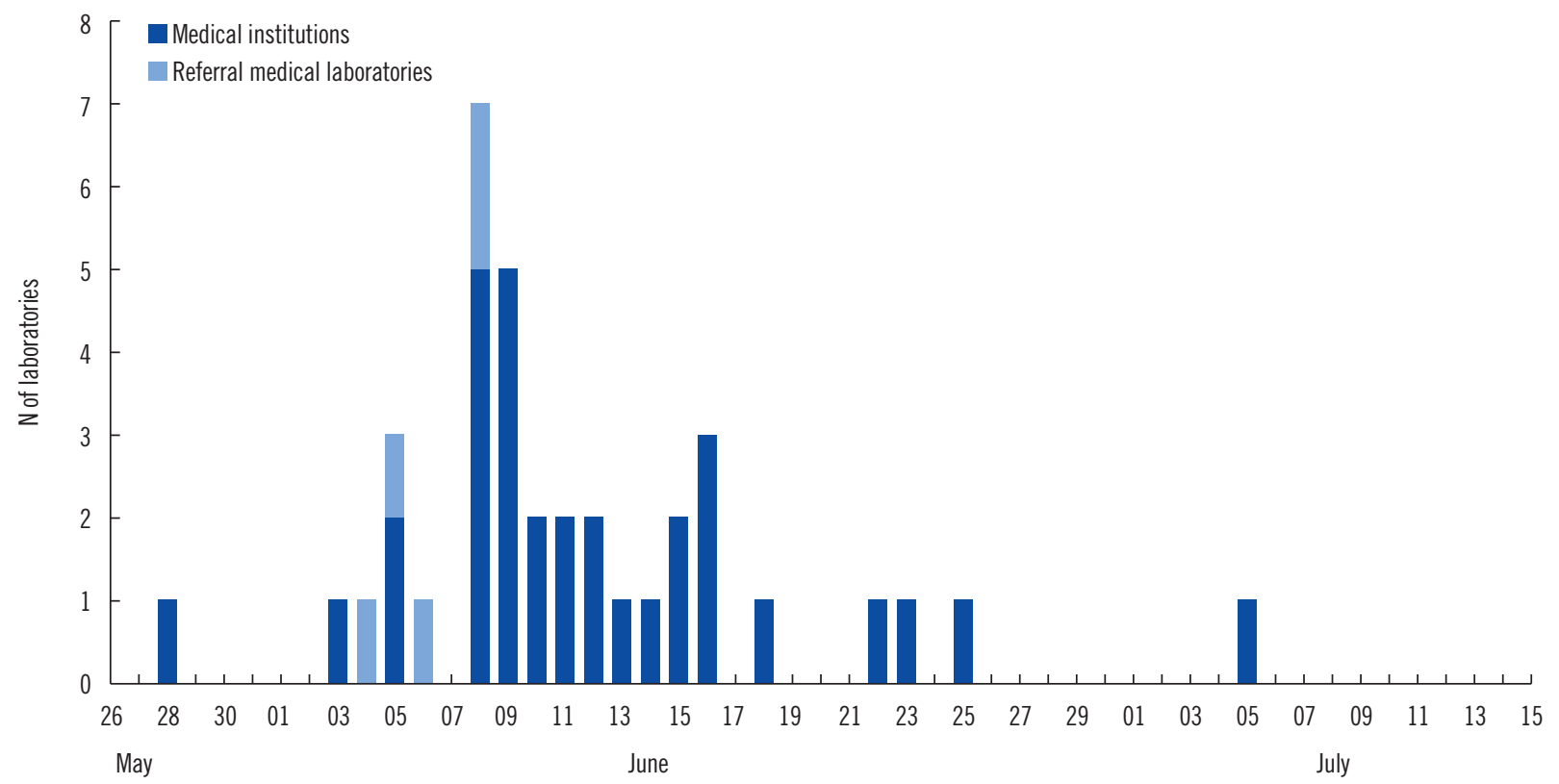

Fig. 2. Number of clinical laboratories that started Middle East respiratory syndrome coronavirus (MERS-CoV) testing in Korea from May 28 to July 15.

tively. The median TAT of more than a half of the laboratories (57.7\%) was less than $6 \mathrm{hr}$. The results were reported within 24 $\mathrm{hr}$ in all medical institutions except one (Table 2).

In the referral medical laboratories, the results were reported in less than 6-9 hr (during weekday daytime) or 9-15 hr (during weekday nights and weekends).

\section{Status of clinical laboratory operation}

Table 2 shows the current status of clinical laboratories in medical institutions with respect to their response to the outbreak of MERS-CoV infections. Many laboratories were able to perform testing throughout the week $(71.4 \%, 25 / 35)$ and ran tests once or twice per day. Specimen was collected mainly by clinicians, and all specimens were directly transported person-to-person. In most cases, clinicians filled out the specimen request information form, and doctors of laboratory medicine completed the test report form. Most of the MERS-CoV rRT-PCR tests were performed only by clinical laboratory technicians of molecular genetics divisions (74.3\% of 35 laboratories). The test results were primarily reported by clinical pathologists via electronic processing. Laboratory biosafety preparedness in response to the MERSCoV outbreak included class II biosafety cabinets (100\%, 35/35); separated pre-PCR, PCR, and post-PCR rooms (88.6\%, 31/35); negative pressure pretreatment rooms $(48.6 \%, 17 / 35)$; and negative pressure sputum collection rooms $(20.0 \%, 7 / 35)$.

As shown in Table 3, the current status of clinical laboratories in referral medical laboratories was similar to that of medical institutions in many aspects. Specimens were transported twice or three times a day.

\section{Pretreatment of specimens, reagents, and equipment}

Many laboratories (75\%, 30/40) used phosphate-buffered saline for pretreatment of sputum specimens. All reagents for the detection of MERS-CoV were components of ready-made kits, and the PowerChek MERS Real-time PCR Kit (KogeneBiotech, Seoul, Korea) was the most commonly used kit (72.5\%, 29/40). Most laboratories used CFX96 (Bio-Rad Laboratories, Hercules, CA, USA) or ABI7500 or 7800 systems (Life Technologies, Carlsbad, CA, USA) for rRT-PCR (85.0\%, 34/40) (Table 4).

\section{DISCUSSION}

The emergence of novel viral pathogens and the evolution of pandemics have presented a new and complex challenge to public health care systems at every level [1, 3, 7, 8]. The outbreak of H1N1 influenza eventually turned out to be relatively mild, despite the fear it engendered as the potential early stage of a pandemic. Breban et al. [9] suggested that MERS-CoV does not yet have pandemic potential. Nevertheless a MERSCoV outbreak recently occurred in the Republic of Korea, and its characteristics were very different from those of the outbreak of H1N1 influenza. The MERS-CoV outbreak was more serious 
Table 2. Turnaround time and status of clinical laboratories in medical institutions

(A) Median turnaround time (TAT) for MERS-CoV testing $(\mathrm{N}=26)$

\begin{tabular}{lrr}
\hline TAT (hr) & N & $\%$ \\
\hline$<6$ & 15 & 57.7 \\
$6-12$ & 8 & 30.8 \\
$12-24$ & 2 & 7.7 \\
$>24$ & 1 & 3.8 \\
Total & 26 & 100.0 \\
\hline
\end{tabular}

(B) Status of clinical laboratories in medical institutions $(\mathrm{N}=35)$

\begin{tabular}{|c|c|c|c|c|c|}
\hline Survey & $\mathrm{N}$ & $\%$ & Survey & $\mathrm{N}$ & $\%$ \\
\hline \multicolumn{3}{|l|}{ Specimen request information form input performed by: } & \multicolumn{3}{|l|}{ Specimen transport } \\
\hline Clinicians & 22 & 62.9 & Person-to-person & 35 & 100.0 \\
\hline Doctors of laboratory medicine & 2 & 5.7 & \multicolumn{3}{|l|}{ Laboratory facilities (plural response) } \\
\hline Infection control nurses & 1 & 2.9 & Negative pressure sputum collection room & 7 & 20.0 \\
\hline No answer & 10 & 28.6 & Negative pressure pretreatment room & 17 & 48.6 \\
\hline Test report form input performed by: & & & Class II biosafety cabinet & 35 & 100.0 \\
\hline Clinicians & 6 & 17.1 & Separated pre-PCR, PCR, and post-PCR rooms & 31 & 88.6 \\
\hline Doctors of laboratory medicine & 11 & 31.4 & \multicolumn{3}{|l|}{ Test performed by: } \\
\hline Technologists of laboratory medicine & 4 & 11.4 & Medical technologists of molecular genetics division & 26 & 74.3 \\
\hline Infection control nurses & 1 & 2.9 & \multirow{2}{*}{$\begin{array}{l}\text { Medical technologists of molecular genetics division } \\
\text { \& other division }\end{array}$} & \multirow[t]{2}{*}{8} & \multirow[t]{2}{*}{22.9} \\
\hline Medical technologists or doctors of laboratory medicine & 3 & 8.6 & & & \\
\hline No answer & 10 & 28.6 & \multicolumn{3}{|l|}{$\begin{array}{l}\text { Medical technologists of molecular genetics division } \\
\text { with newly employed technologists for MERS-CoV test }\end{array}$} \\
\hline Operating days of MERS-CoV test & 5 & 143 & \multicolumn{3}{|l|}{ Results reported by: } \\
\hline Mon-Fri & 5 & 14.3 & Doctors of laboratory medicine - Faculty & 29 & 82.9 \\
\hline Mon-Sun & 35 & $74.3)$ & \multirow{3}{*}{$\begin{array}{l}\text { Doctors of laboratory medicine - Fellow } \\
\text { Doctors of laboratory medicine - Faculty \& fellow } \\
\text { Doctors of laboratory medicine - Faculty, fellow, } \\
\text { \& resident }\end{array}$} & 3 & 8.6 \\
\hline Onerating hours of MERS-CoV test & $2 v$ & 71.4 & & 2 & 5.7 \\
\hline Regular working hours & 20 & 57.1 & & 1 & 2.9 \\
\hline Overtime & 2 & 5.7 & \multicolumn{3}{|l|}{ Form of the report } \\
\hline $24 \mathrm{hr}$ & 13 & 37.1 & Electronic medical record, group SMS \& phone call & 2 & 5.7 \\
\hline Number of MERS-CoV tests run per day & & & Electronic medical record \& group SMS & 6 & 17.1 \\
\hline One & 16 & 45.7 & Electronic medical record, report paper \& phone call & 1 & 2.9 \\
\hline Two & 9 & 25.7 & Electronic medical record \& report paper & 1 & 2.9 \\
\hline More than three & 5 & 14.3 & Electronic medical record \& phone call & 5 & 14.3 \\
\hline Others* & 5 & 14.3 & \multirow[t]{7}{*}{ Electronic medical record } & \multirow[t]{7}{*}{20} & \multirow[t]{2}{*}{57.1} \\
\hline Specimen collections performed by: & & & & & \\
\hline Clinicians & 18 & 51.4 & & & \\
\hline Nurses & 2 & 5.7 & & & \\
\hline Clinicians \& nurses & 11 & 31.4 & & & \\
\hline Clinicians, nurses \& medical technologists & 1 & 2.9 & & & \\
\hline No answer & 3 & 8.6 & & & \\
\hline
\end{tabular}

${ }^{*}$ Others, dependent on specimen reception or situation.

Abbreviations: MERS-CoV, Middle East respiratory syndrome coronavirus; N, Number of medical institutions; SMS, short message service. 
Table 3. Status of clinical laboratories in referral medical laboratories $(\mathrm{N}=5)$

\begin{tabular}{|c|c|c|}
\hline Survey & $\mathrm{N}$ & $\%$ \\
\hline \multicolumn{3}{|l|}{ Operating days of MERS-CoV tests } \\
\hline Mon-Sun & 5 & 100.0 \\
\hline \multicolumn{3}{|l|}{ Operating hours of MERS-CoV tests } \\
\hline $24 \mathrm{hr}$ & 5 & 100.0 \\
\hline \multicolumn{3}{|l|}{ Number of MERS-CoV tests per day } \\
\hline Two & 3 & 60.0 \\
\hline Three & 1 & 20.0 \\
\hline Three on weekdays \& two on weekends & 1 & 20.0 \\
\hline \multicolumn{3}{|l|}{ Specimen transport } \\
\hline \multicolumn{3}{|l|}{ Transported by: } \\
\hline Referral medical laboratories themselves & 3 & 60.0 \\
\hline Commercial transport services & 1 & 20.0 \\
\hline Both & 1 & 20.0 \\
\hline With refrigerated system (vehicles) & 2 & 40.0 \\
\hline With icepack & 5 & 100.0 \\
\hline \multicolumn{3}{|l|}{ Protection for transport agents } \\
\hline N-95 mask \& disposable gloves & 4 & 80.0 \\
\hline N-95 mask, disposable gloves, \& disposable gown & 1 & 20.0 \\
\hline \multicolumn{3}{|l|}{ Number of specimen transportations per day } \\
\hline Two & 2 & 40.0 \\
\hline Three & 2 & 40.0 \\
\hline Three on weekdays \& two on weekends & 1 & 20.0 \\
\hline \multicolumn{3}{|l|}{ Laboratory facilities (plural response) } \\
\hline Negative pressure pretreatment room & 4 & 80.0 \\
\hline Class II biosafety cabinet & 5 & 100.0 \\
\hline Separated pre-PCR, PCR, and post-PCR rooms & 5 & 100.0 \\
\hline \multicolumn{3}{|l|}{ Test performed by: } \\
\hline Medical technologists of molecular genetics division & 3 & 60.0 \\
\hline $\begin{array}{l}\text { Medical technologists of molecular genetics division } \\
\text { \& other division }\end{array}$ & 2 & 40.0 \\
\hline \multicolumn{3}{|l|}{ Results reported by: } \\
\hline Doctors of laboratory medicine-Faculty & 5 & 100.0 \\
\hline \multicolumn{3}{|l|}{ Form of report } \\
\hline Electronic medical records & 4 & 80.0 \\
\hline Phone call \& report paper & 1 & 20.0 \\
\hline
\end{tabular}

Abbreviation: MERS-CoV, Middle East respiratory syndrome coronavirus.

than expected, and numerous problems arose concerning infection control and prevention in hospitals and among the general public.

Because clinical laboratories are usually on the front lines for
Table 4. Pretreatment, reagents, and equipment for MERS-CoV testing in 2015 outbreak in Korea

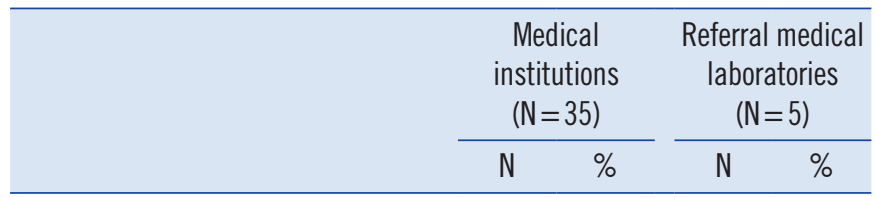

Pretreatment of sputum

\begin{tabular}{lrrrr} 
PBS & 25 & 71.4 & 5 & 100.0 \\
NALC & 3 & 8.6 & & \\
Commercial lysis kits & 2 & 5.7 & & \\
DW & 1 & 2.9 & & \\
PBS \& NALC & 1 & 2.9 & & \\
No pretreatment & 1 & 2.9 & & \\
No answer & 3 & 5.7 & & \\
Reagents & & & & \\
KogeneBiotech & 20 & 57.1 & 5 & 100.0 \\
SolGent & 6 & 17.1 & & \\
Bioneer & 4 & 11.4 & & \\
NANOBIOSYS & 1 & 2.9 & & \\
KogeneBiotech \& Bioneer & 1 & 2.9 & & \\
KogeneBiotech \& NANOBIOSYS & 1 & 2.9 & & \\
KogeneBiotech \& Seegene & 1 & 2.9 & & \\
KogeneBiotech, SolGent, \& NANOBIOSYS & 1 & 2.9 & & \\
Equipment & & & & \\
BIO-RAD CFX96 & 15 & 42.9 & 1 & 20.0 \\
ABI7500 or 7500 Fast & 11 & 31.4 & 4 & 80.0 \\
Bioneer Exicycler & 4 & 11.4 & & \\
LG SLAN & 1 & 2.9 & & \\
NANOBIOSYS G2-4 & 1 & 2.9 & & \\
BI0-RAD CFX96 \& Bioneer Exicycler & 1 & 2.9 & & \\
BIO-RAD CFX96, ABI7500 or 7800 & 1 & 2.9 & & \\
$\quad$ \& NANOBIOSYS G2-4 & & & & \\
No answer & 1 & 2.9 & & \\
\hline Ab & &
\end{tabular}

Abbreviations: MERS-CoV, Middle East respiratory syndrome coronavirus; PBS, phosphate-buffered saline; NALC, N-acetyl L-cysteine; DW, deionized water; KogeneBiotech, KogeneBiotech PowerChek MERS-CoV real-time PCR kit; SolGent, SolGent DiaPlexQ MERS-CoV virus detection kit; Bioneer, Bioneer AccuPower MERS-CoV real-time RT-PCR kit; NANOBIOSYS, NANOBIOSYS MERS-CoV corona virus detection kit; BIO-RAD CFX96, BIORAD CFX96 real-time PCR system; ABI7500 or 7500 Fast, Life Technologies ABI7500 or 7800 real-time PCR system; Bioneer Exicycler, Bioneer Exicycler 96 real-time PCR system; LG SLAN, LG Life Sciences SLAN real-time PCR system; NANOBIOSYS G2-4, NANOBIOSYS real-time PCR G2-4.

the detection of emerging pathogens, the ability of these laboratories to respond to an outbreak is critical for infection control and prevention. The elements of clinical laboratory preparedness and responsiveness include availability of personal protec- 
tive equipment and its appropriate use, availability and use of appropriate testing supplies, adequacy of staffing, and infrastructure requirements including laboratory space [2]. In 2012 and 2013, the European Centre for Disease Prevention and Control (ECDC) and the WHO Regional Office for Europe conducted a joint survey [10]. Although the number of countries that had laboratory capabilities to detect and confirm MERSCoV increased in 2013 (55.8\%, 29 of 52 countries) compared with 2012 (47.8\%, 22 of 46 countries), a half of the countries were still unable to test MERS-CoV [10].

The present study reveals a snapshot of the current status and capability of clinical laboratories to respond to the MERSCoV outbreak in the Republic of Korea. Most clinical laboratories participating in the survey were considered to have sufficient capacity to respond to the outbreak. Immediately after the MERS-CoV rRT-PCR test was approved, many laboratories were able to perform testing seven days a week and the number of tests reported increased dramatically. The median TAT of more than a half of the laboratories (57.7\%) was shorter than $6 \mathrm{hr}$, and the minimum median TAT was $4.00 \mathrm{hr}$. The results were reported within $24 \mathrm{hr}$ in all medical institutions except one. One limitation of our study is that even though we obtained responses from the majority of medical institutions and referral medical laboratories, the results may not be fully representative for a number of reasons. Data were collected before the end of the MERS-CoV outbreak and did not include some major medical institutions and public health laboratories such as the Korea Centers for Disease Control and Prevention. In addition, our results report the number of MERS-CoV rRT-PCR tests, and not the number of MERS-CoV cases.

Previously, Sousa et al. [11] suggested that the use of upper respiratory specimens (e.g., nasopharyngeal swabs) for MERSCoV diagnosis might not be as sensitive as the use of lower respiratory tract specimens. The laboratory diagnostic guidelines for MERS-CoV testing of the Korean Society for Laboratory Medicine recommended using specimens from the lower respiratory tract. In the present survey, the number of positive results from MERS-CoV rRT-PCR was significantly higher in specimens obtained from the lower respiratory tract $(1.01 \%, 204 / 20,183)$ compared with specimens from the upper respiratory tract $(0.6 \%, 41 / 6,785)(P=0.002$, chi-square test). Therefore, the specimen type is expected to have a significant impact on diagnostic sensitivity and should be considered when testing emerging pathogens.

For laboratory biosafety in response to the MERS-CoV outbreak, class II biosafety cabinets were adequately supplied but high-end engineering facilities such as negative pressure sputum collection and pretreatment rooms were not readily available. However, no laboratory-acquired infections were reported during the outbreak, thus standard precautions with droplet precautions appeared to be sufficient for the prevention of laboratory-acquired infection of MERS-CoV. Nonetheless, improvements in engineering laboratory biosafety are needed for preparedness to test agents with a higher biosafety level.

Clinical laboratories have the primary responsibility for testing specimens to support clinical decision-making. Although public health laboratories also test specimens to aid clinical decisions, their roles in surveillance, strain identification, and tracking of drug resistance are arguably their main priorities [12]. Moreover, clinical laboratories often have resources available that allow for rapid expansion [12]. In conclusion, the results of this survey contribute to the comprehensive view of clinical laboratory response in the Republic of Korea to the recent outbreak of 2015 MERS-CoV. On the basis of currently available data, clinical laboratories in Korea were able to expand their diagnostic capacity in a short time and achieve a TAT of shorter than nine hours with testing seven days per week to response to the recent MERSCoV outbreak, although the delay in the early period of the outbreak should be improved. Therefore, clinical laboratories should be ready for the maintenance and enhancement of laboratory responses in preparation for future emerging infections.

\section{Authors' Disclosures of Potential Conflicts of Interest}

No potential conflicts of interest relevant to this article were reported.

\section{Acknowledgments}

Many thanks to the directors and clinical pathologists in the medical institutions and referral medical laboratories: Young Ae Lim, II Joong Park (Ajou University Hospital); Seog-Woon Kwon, Heungsup Sung (ASAN Medical Center); Seong Geun Hong (CHA Bundang Medical Center); Seung-Jung Kee (Cheonnam National University Hospital); Kyeong Seob Shin, Seong Tae Maeng (Chonbuk National University Hospital); Young Joo Cha, Mi-Kyung Lee (Chung-Ang University Hospital); Sun-hoe Koo, Jimyung Kim (Chungnam National University Hospital); Jong Wan Kim (Dankook University Hospital); Hyun Soo Kim (Hallym University Dongtan Sacred Heart Hospital); Gwiyoung Oh, Jin-Hee Cho (EONE Laboratories); Chunhwa Ihm (Eulji University Hospital); 
Jungwon Huh, Miae Lee (Ewha Womans University Mokdong Hospital); Seok Hoon Jeong (Gangnam Severance Hospital); Eun Hee Lee, Hee-Jung Kim (Green Cross Laboratories); Jeong Seon Yang (H Plus Yang Ji Hospital); Jeong Hwan Shin (Inje University Busan Paik Hospital); Tae Hee Han, Bo-Moon Shin (Inje University Sanggye Paik Hospital); Hee-Yeon Woo, Min-Jung Kwon (Kangbuk Samsung Hospital); Ji-Young Park, Jae-Seok Kim (Hallym University Kangdong Sacred Heart Hospital); Wonkeun Song (Hallym University Kangnam Sacred Heart); Namhee Ryoo (Keimyung University Dongsan Medical Center); Kyung So Jeon (KEPCO Medical Center); Younjung Cho (Korea University Anam Hospital); Chae Seung Lim (Korea University Guro Hospital); Young Ah Kim, Jong-ha Yoo (National Health Insurance Service Ilsan Hospital); Hyung Hoi Kim, Jongyoun Yi (Pusan National University Hospital); Chulhun L. Chang, In-Suk Kim (Pusan National University Yangsan Hospital); Hyun-Young Chi, Nae Yu (Samkwang Medical Laboratories); Eun-Suk Kang, Chang-Seok Ki (Samsung Medical Center); Sun Hwa Lee, Kyoung Ho Roh (Seegene Medical Foundation); Ki Ho Hong (Seoul Medical Center); Young Jin Kim, Ah Ra Cho (Seoul Medical Science Institute); Junghan Song, Kyoung Un Park (Seoul National University Bundang Hospital); Sung Sup Park, Moon-Woo Seong (Seoul National University Hospital); Jeong-Ho Kim, Dongeun Yong (Severance Hospital); Sue Shin, Soo Hyun Seo (SMG-SNU Boramae Medical Center); You Kyoung Lee, Hee Bong Shin (Soonchunhyang University Bucheon Hospital); Jeong Won Shin (Soonchunhyang University Hospital Seoul); Hae Kyung Lee (The Catholic University of Korea Uijeongbu St. Mary's Hospital); Jehoon Lee (The Catholic University of Korea Yeouido St. Mary's Hospital).

\section{REFERENCES}

1. Cowling BJ, Park M, Fang VJ, Wu P, Leung GM, Wu JT. Preliminary epidemiological assessment of MERS-CoV outbreak in South Korea, May to June 2015. Euro Surveill 2015;20:7-13.

2. Hayden RT, Wick MT, Rodriguez AB, Caliendo AM, Mitchell MJ, Ginocchio CC. A survey-based assessment of United States clinical laboratory response to the 2009 H1N1 influenza outbreak. Arch Pathol Lab Med 2010;134:1671-8.

3. Sullivan SJ, Jacobson RM, Dowdle WR, Poland GA. 2009 H1N1 influenza. Mayo Clin Proc 2010;85:64-76.

4. Crawford JM, Stallone R, Zhang F, Gerolimatos M, Korologos DD, Sweetapple C, et al. Laboratory surge response to pandemic (H1N1) 2009 outbreak, New York City metropolitan area, USA. Emerg Infect Dis 2010;16:8-13.

5. Lautenbach E, Saint S, Henderson DK, Harris AD. Initial response of health care institutions to emergence of H1N1 influenza: experiences, obstacles, and perceived future needs. Clin Infect Dis 2010;50:523-7.

6. Lee WG, Kwak YS, Lee DH, Hwang YS, Lee KN. Clinical pathology laboratory inspection and accreditation in Korea I: Development of the system and its trial. Korean J Clin Pathol 2001;21:86-92.

7. Oboho IK, Tomczyk SM, Al-Asmari AM, Banjar AA, Al-Mugti H, Aloraini MS, et al. 2014 MERS-CoV outbreak in Jeddah--a link to health care facilities. N Engl J Med 2015;372:846-54.

8. Tartari E, Allegranzi B, Ang B, Calleja N, Collignon P, Hopman J, et al. Preparedness of institutions around the world for managing patients with Ebola virus disease: an infection control readiness checklist. Antimicrob Resist Infect Control 2015;4:22.

9. Breban R, Riou J, Fontanet A. Interhuman transmissibility of Middle East respiratory syndrome coronavirus: estimation of pandemic risk. Lancet 2013;382:694-9.

10. Pereyaslov D, Rosin P, Palm D, Zeller H, Gross D, Brown CS, et al. Laboratory capability and surveillance testing for Middle East respiratory syndrome coronavirus infection in the WHO European Region, June 2013. Euro Surveill 2014;19:20923.

11. de Sousa R, Reusken C, Koopmans M. MERS coronavirus: data gaps for laboratory preparedness. J Clin Virol 2014;59:4-11.

12. Meltzer MI, McNeill KM, Miller JD. Laboratory surge capacity and pandemic influenza. Emerg Infect Dis 2010;16:147-8. 